\title{
Reflections on and Extensions of the Fuller and Tabor Theory of Rough Surface Adhesion
}

\author{
J. A. Greenwood ${ }^{1}$
}

Received: 28 August 2017 / Accepted: 11 October 2017 / Published online: 2 November 2017

(C) The Author(s) 2017. This article is an open access publication

\begin{abstract}
The classical Fuller and Tabor theory of rough surface adhesion is reviewed, and its limited applicability noted. New results using an extension of the JKR adhesion theory to include the effect of van der Waals adhesion forces are described. These show the influence of the Tabor parameter $\mu$ : moderate values of $\mu(\mu<100)$ lead to increased forces during loading, and so are found to predict much lower hysteresis losses. A further extension is to replace the GW surface roughness model used by Fuller and Tabor by a model based on the Nayak model of a surface as a random field, as in the Bush, Gibson and Thomas (BGT) theory of non-adhesive contact. The results are qualitatively the same, and the differences may well be largely due to the necessary redefinition of the adhesion index using the rms profile curvature in place of the asperity radius of curvature used in the GW model. `
\end{abstract}

Keywords Adhesion · Surface roughness · Fuller and Tabor $\cdot$ JKR theory $\cdot$ Tabor parameter $\cdot$ Nayak parameter

\section{Fuller and Tabor's Analysis of Adhesion of Rough Surfaces}

Fuller and Tabor's classic analysis of the effect of surface roughness [1] on adhesion combines the Greenwood and Williamson (GW) model of surface roughness [2], in which the roughness is represented by an array of paraboloidal

J. A. Greenwood jag@eng.cam.ac.uk

1 Department of Engineering, University of Cambridge, Trumpington Street, Cambridge CB2 1PZ, UK asperities, with the JKR theory of the adhesive contact between a single elastic sphere and a plane [3].

In the GW model, the summits are of fixed curvature $(1 / R)$ and have a Gaussian height distribution $\phi(z)=\frac{1}{\sigma \sqrt{2 \pi}} \exp \left(-z^{2} / 2 \sigma^{2}\right)$ : we shall throughout work with non-dimensional heights $\varsigma \equiv z / \sigma$, so that the height distribution becomes $\phi(\varsigma)=\frac{1}{\sqrt{2 \pi}} \exp \left(-\varsigma^{2} / 2\right)$.

The JKR theory gives equations linking the load and the deformation with the contact radius, from which the load $P$ may be found from the deformation $\delta$ in the form $P=f(\delta)$. Fuller and Tabor use the non-dimensional variables introduced by Maugis [4] (which lead to particularly simple forms for the JKR equations), but we prefer to omit Maugis' simplifying numerical factors and define

$\hat{P}=\frac{P}{R \Delta \gamma} ; \quad \hat{\delta}=\left(\frac{E^{* 2}}{R \Delta \gamma^{2}}\right)^{1 / 3} \delta ; \quad \hat{a}=\left(\frac{E^{*}}{R^{2} \Delta \gamma}\right)^{1 / 3} a$.

More compactly: $\hat{P}=\frac{P}{R \Delta \gamma} ; \hat{\delta}=\beta^{2} \frac{\delta}{R} ; \hat{a}=\beta \frac{a}{R}$ where $\beta \equiv\left(\frac{E^{*} R}{\Delta \gamma}\right)^{1 / 3}$.

Using these variables, the JKR equations become:

$\hat{\delta}=\hat{a}^{2}-\sqrt{2 \pi \hat{a}} ; \quad \hat{P}=(4 / 3) \hat{a}^{3}-\sqrt{8 \pi \hat{a}^{3}}$.

Here, $\delta$ is again a compression, and $\hat{P}$ a compressive force, as in the GW model: these are the equations introduced by Ciavarella et al. [5], but with the opposite (earlier) sign convention.

It will be recalled that, according to the JKR theory, there is no interaction between the two surfaces until contact is made, when the contact radius immediately jumps to $\hat{a}_{A}=(2 \pi)^{1 / 3}$ and the force to $\hat{P}_{A}=-4 \pi / 3$; separation (under 
"fixed grips" conditions) occurs when $\tilde{\delta}_{c}=-(3 / 4) \pi^{2 / 3}$ (with $\left.\hat{a}_{c}=(1 / 2) \pi^{1 / 3}, \hat{P}_{c}=-(5 / 6) \pi\right)$.

For a GW surface with $\mathrm{N}$ asperities which have a height distribution $\phi(\varsigma)$, the contact force for a mean plane separation $h \equiv d / \sigma$ will be

$$
\begin{aligned}
\hat{P}(h) & =N \int_{-\infty}^{\infty} f(\hat{\delta}) \phi(\varsigma) \mathrm{d} \varsigma \quad \text { where } \hat{\delta}=\beta^{2}(\langle z-d\rangle / R) \\
& =\left(\beta^{2} \sigma / R\right)\langle\varsigma-h\rangle \equiv\langle\varsigma-h\rangle / Z
\end{aligned}
$$

where $\langle\ldots\rangle$ are Macaulay brackets, i.e. set to zero when the content is negative, ${ }^{1}$ and $Z \equiv R /\left(\beta^{2} \sigma\right)$

Now change the integration variable to $\hat{\delta}$. Then $\varsigma=h+Z \hat{\delta}$, and the load becomes

$\hat{P}(h)=N Z \int_{-\infty}^{\infty} f(\hat{\delta}) \phi(h+Z \hat{\delta}) \mathrm{d} \hat{\delta}$.

Assuming, in accordance with the JKR theory, that asperities do not jump into contact, then during loading we have $f(\hat{\delta})=0$ for $\hat{\delta}<0$. The lower limit becomes zero, and

$$
\begin{aligned}
\hat{P}(h) & =N Z \int_{0}^{\infty} f(\hat{\delta}) \phi(h+Z \hat{\delta}) \mathrm{d} \hat{\delta} \\
& =\frac{N Z}{\sqrt{2 \pi}} \int_{0}^{\infty} f(\hat{\delta}) \exp \left[-(h+Z \hat{\delta})^{2} / 2\right] \mathrm{d} \hat{\delta}
\end{aligned}
$$

The parameter $Z \equiv R /\left(\beta^{2} \sigma\right) \equiv\left(R \Delta \gamma^{2} / E^{* 2} \sigma^{3}\right)^{1 / 3}$ is Fuller and Tabor's adhesion index $\Delta_{c}$, but with their numerical factor $(3 / 4) \pi^{2 / 3} \approx 1.609$ removed to agree with the present nondimensional variables, so that $Z=0.6216 \Delta_{c}$ : we shall refer to $Z$ as the adhesion index. ${ }^{2}$

Figure 1 brings out the embarrassing feature of the F\&T model: it is not easy to reach the zero load point without pushing the Greenwood and Williamson surface roughness model too far: the idea of individual, non-interacting, paraboloidal asperities extending down below the asperity mean plane is absurd. While an experimenter might possibly be content to place his specimens together under zero load and then attempt to separate them, without insisting on applying a positive load, he will certainly not be satisfied with less. So with the rather generous restriction of requiring only that $h>0$, we cannot trust the detailed predictions of the theory for values of the adhesion index above $Z=0.45$ (accurately $0.456)$.

We also encounter a second barrier: for when $Z<0.2$, there is very little adhesion to study!

A careful investigation confirms that there is always a net adhesive force at sufficiently high separations, so that

\footnotetext{
1 A control engineer would write $x H(x)$ where we write $\langle x\rangle$.

${ }^{2} Z$ is $\theta$ as used by Maugis: but F\&T use $\theta \equiv Z^{-3 / 2}$ as their alternative adhesion index.
}

surfaces will always jump into contact under zero load. A small, but positive, force will be required to separate them.

As an example, for $Z=0.15$, the zero load point is $h_{c}=+3.52$ and the maximum tensile force during loading is $3 \times 10^{-5} N R \Delta \gamma$ : separating the surfaces (see below) requires $6 \times 10^{-4} N R \Delta \gamma$, both presumably, in practice, undetectable.

\subsection{Unloading}

During unloading it is no longer true that $f(\hat{\delta})=0$ when $\hat{\delta}<0$. For during unloading, all the contacts formed during loading will remain in contact until $\hat{\delta}=\hat{\delta}_{c} \equiv-(3 / 4) \pi^{2 / 3}$. For unloading from $\varsigma=h_{1}$, the lower limit then becomes either $\hat{\delta}=\left(h_{1}-h\right) / Z$ or $\hat{\delta}=\hat{\delta}_{c}$, and the higher one (NB both are negative!) must be chosen. Thus, Eq. (4) is replaced by (Fig. 2)

$\hat{P}=\frac{N Z}{\sqrt{2 \pi}} \int_{\hat{\delta}_{0}}^{\infty} f(\hat{\delta}) \exp \left[-\{h+Z \hat{\delta}\}^{2} / 2\right] \mathrm{d} \hat{\delta}$

where $\hat{\delta}_{0}=\max \left\{\left(h_{1}-h\right) / Z, \hat{\delta}_{c}\right\}$.

As Fuller and Tabor found, when the JKR adhesion theory is used, the unloading curve is very different from tha loading curve: there is a substantial hysteresis energy loss.

\section{Extension of the Fuller and Tabor Theory: Inclusion of Jumping-On}

The JKR theory, despite its neglect of van der Waals attractive forces, is known to give an excellent description of the behaviour once contact is established, provided the value of the Tabor parameter ${ }^{3} \mu \equiv\left(\frac{R \Delta \gamma^{2}}{E^{* 2} \varepsilon^{3}}\right)^{1 / 3}$ exceeds 5 .

But the absence of any interaction before contact takes place is an unfortunate feature of the JKR theory. Indeed, perhaps the best established part of the theory of the interaction between solids is the presence of van der Waals forces, attracting the surfaces across a gap $h$ with a force/ unit area inversely proportional to the cube of the distance between them. Full numerical analyses of elastic contact between two spheres using the law of force $\sigma=\frac{8 \Delta y}{3 \varepsilon}\left[\frac{\varepsilon^{3}}{h^{3}}-\frac{\varepsilon^{9}}{h^{9}}\right]$ (based on the Lennard-Jones 6-12 potential law between molecules) [7-10] lead to results such as shown in Fig. 3. We note the good general agreement between the numerical results and the JKR curve, but the regrettable absence of pre-contact interactions in the JKR theory: there is nothing corresponding to the loading branch PA.

\footnotetext{
${ }^{3}$ Introduced by Tabor [6], modified by [7, 8].
} 
Fig. 1 Loading curves. The region $h<0$ has been shaded to indicate that the GW model should not be pushed to negative separations. Then for $Z \geq 0.5$, on this model zero load cannot be reached. For $Z \leq 0.2$ , although the initial contact is always tensile, its magnitude is very small

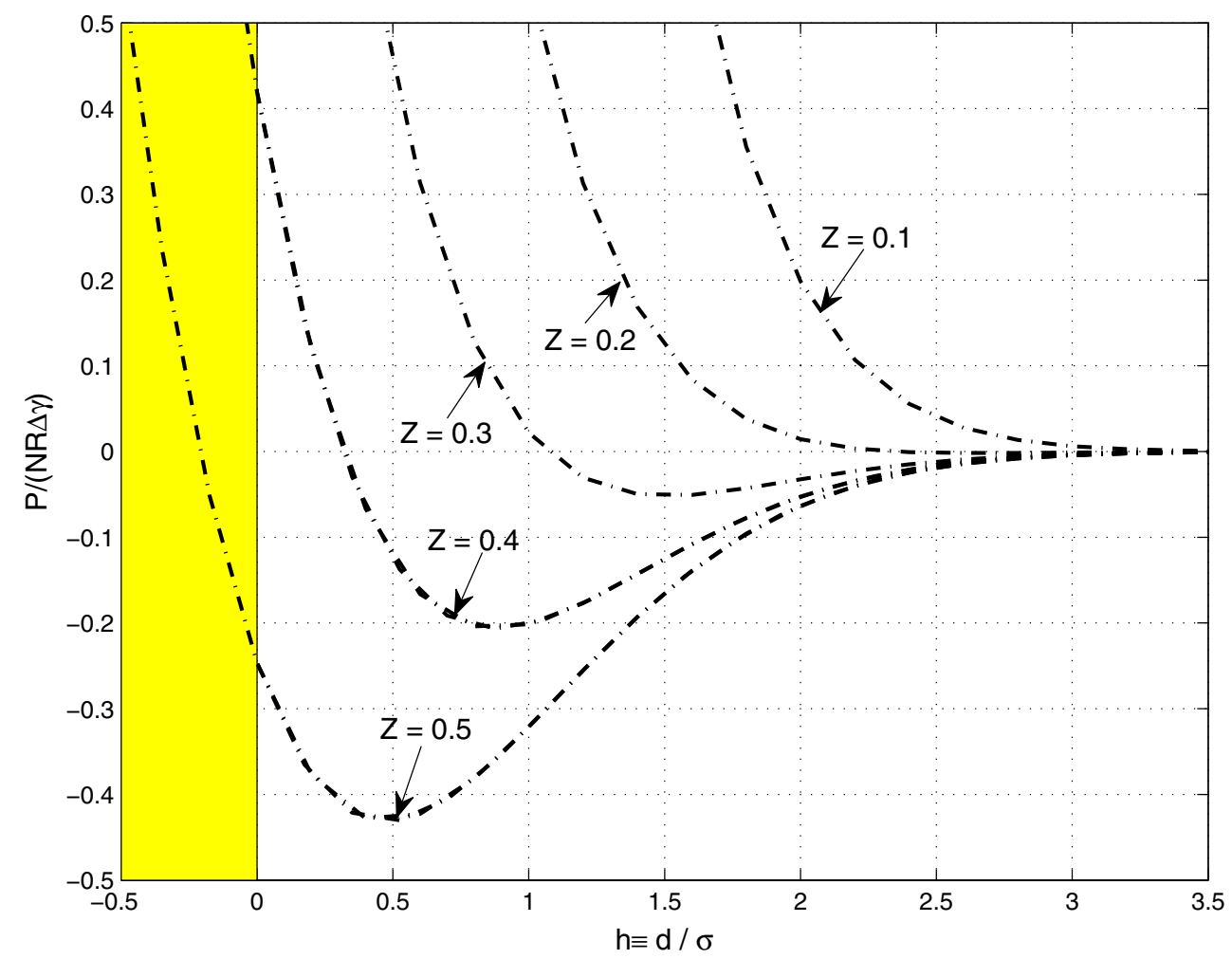

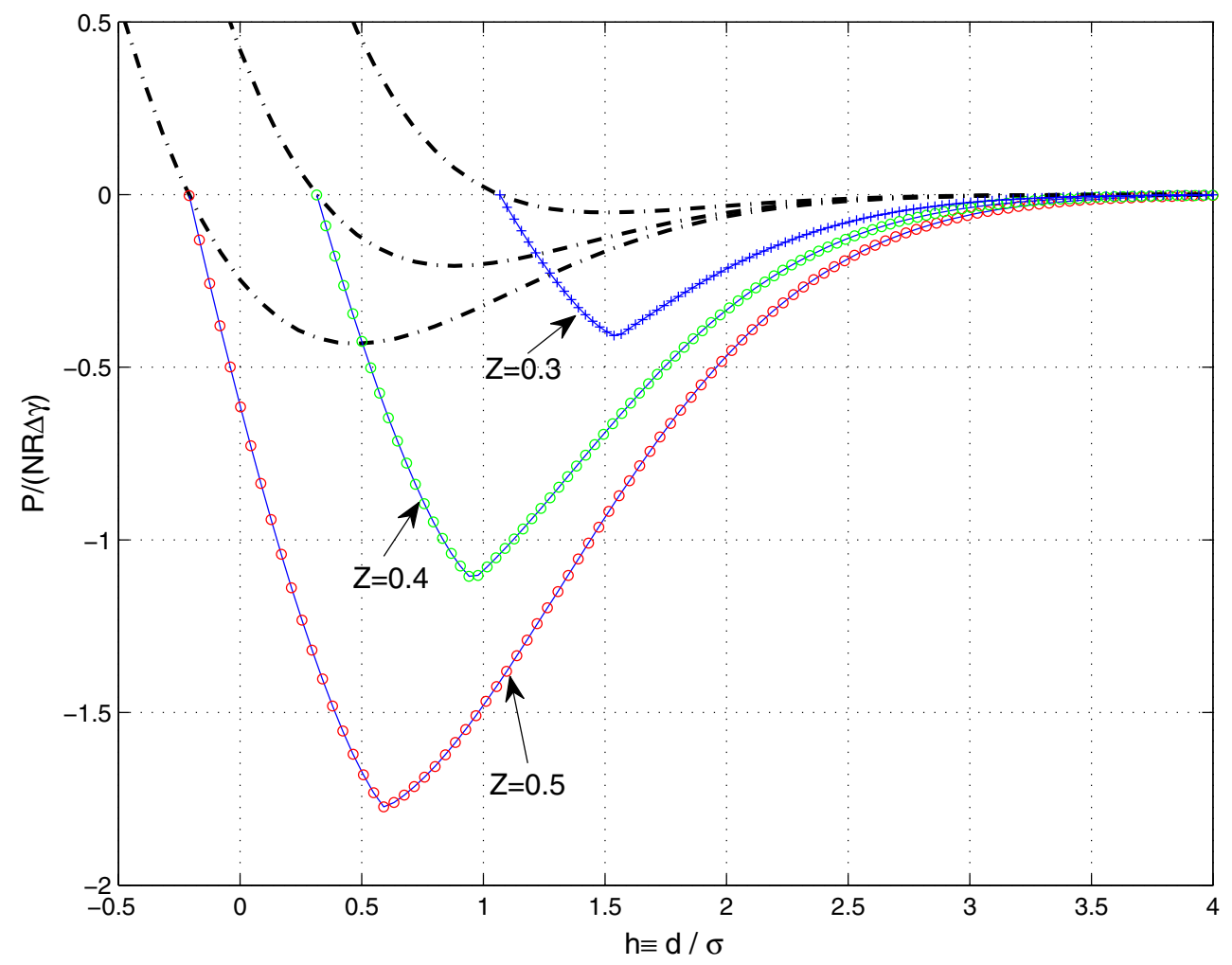

Fig. 2 Unloading from zero load. The dashed lines are the loading curves. Note the enormous difference between loading and unloading 


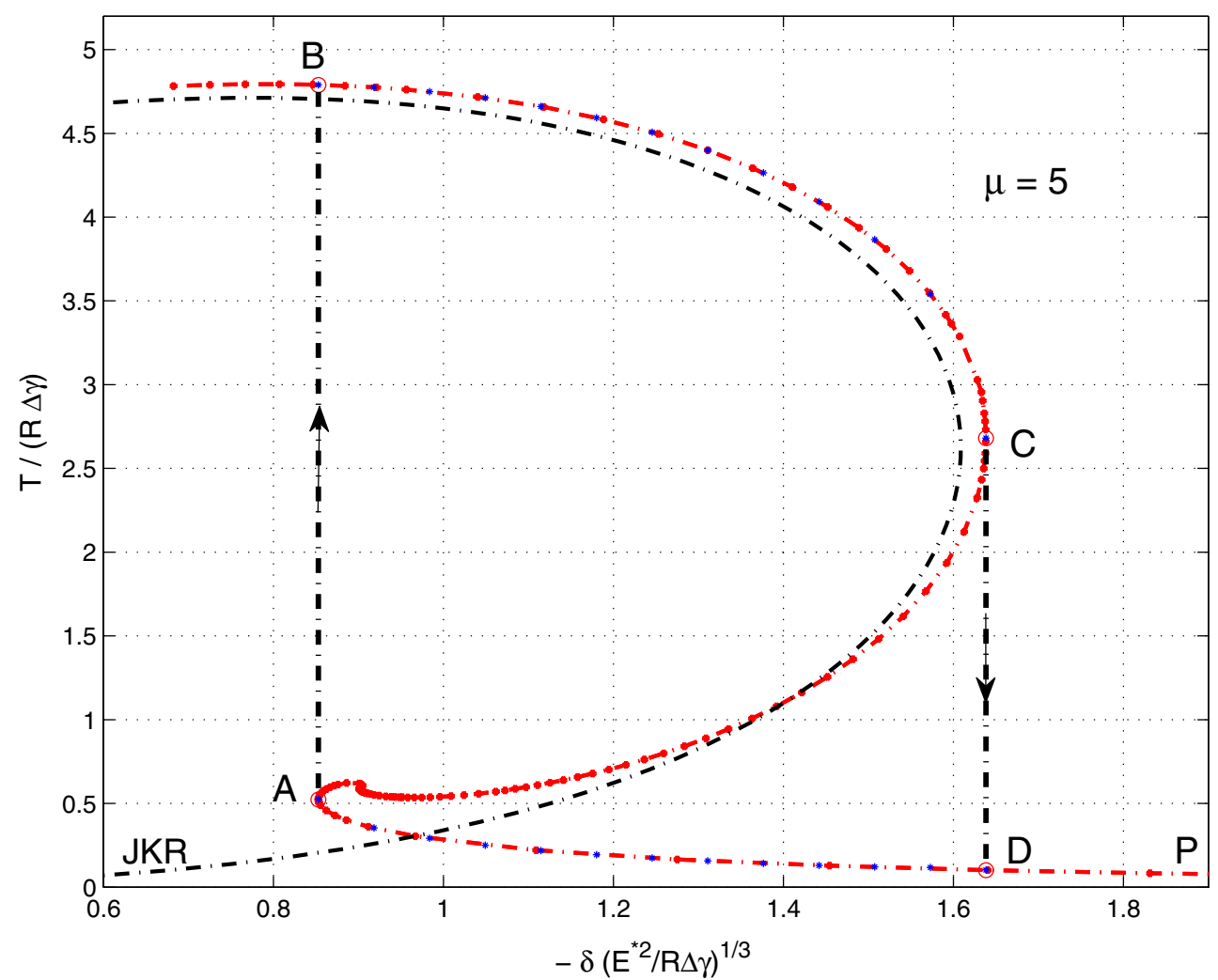

Fig. 3 Comparison of JKR theory with a numerical solution using Lennard-Jones surface forces. After the initial lead-in PA, there is good agreement between the two for $\mu=5$, and this becomes better as $\mu$ increases. For an individual contact, jumping-on is a sudden

Thus, while on the JKR theory jumping-on takes place only when $\delta=0$, the numerical solution places it much earlier, at point A: which here, for $\mu=5$, is at $-\hat{\delta} \approx 0.86$. (In contrast, jumping-off, at point $\mathrm{C}$, occurs reasonably near to the JKR prediction.) Now a careful study of the lower branch of the numerical solution, from $\mathrm{P}$ to $\mathrm{A}$, shows that here the repulsive term in the surface force law plays a negligible part. Wu [11] developed an elegant dimensionless formulation of the elastic contact problem when only the van der Waals term is included, from which he showed that the turning point B will be at $\hat{\delta}_{A}=\frac{1}{\mu}-\frac{2.641}{\mu^{4 / 7}}$, where only the numerical factor depends on a numerical solution. (For a detailed discussion of this result, and of its accuracy, see Ciavarella et al. [5], but summarising, the neglect of the compressive term, already reasonable at $\mu=5$, becomes increasingly more correct as $\mu$ increases.)

Ciavarella et al. also argue that jumping-on, to point B, can be taken as jumping-on to the corresponding point of the JKR curve. We therefore propose to extend the Fuller and Tabor analysis by assuming that during loading, there will be no interaction until $\hat{\delta}=\hat{\delta}_{A} \equiv \frac{1}{\mu}-\frac{2.641}{\mu^{4 / 7}}$, but the contact force will then jump to the point on the JKR curve $\hat{P}=f(\hat{\delta})$ increase in the adhesive force with no change in position: in the JKR theory model it takes place only at contact $(\delta=0)$. Van der Waals forces make jumping-on occur much earlier. Of course experimentally the supporting cantilever means that both $P$ and $\delta$ change

close to point B. Thus, we take the force during loading to be given by

$\hat{P}=\frac{N Z}{\sqrt{2 \pi}} \int_{\hat{\delta}_{A}}^{\infty} f(\hat{\delta}) \exp \left[-\{h+Z \hat{\delta}\}^{2} / 2\right] \mathrm{d} \hat{\delta}$.

Figure 4 shows the large effect that early jumping-on has on the loading curve. For $\mu=5$, usually regarded as justifying the use of the JKR equations, the greatest tensile force, during loading, is increased by almost a factor 10 .

However, the real interest is in the behaviour during unloading, and in particular the value of the pull-off force. For unloading, Eq. (5) also needs to be altered. All the contacts made during loading will remain in contact until the critical extension $\hat{\delta}_{\mathrm{C}} \equiv-(3 / 4) \pi^{2 / 3}$ is reached, so the lower limit in the integral of Eq. (5) must be changed from $\hat{\delta}_{0}=\max \left(\left\{\left(h_{1}-h\right) / Z\right\}, \hat{\delta}_{\mathrm{C}}\right)$ to $\hat{\delta}_{0}=\max \left(\left\{\left(h_{1}-h\right) / Z+\hat{\delta}_{\mathrm{A}}\right\}, \hat{\delta}_{\mathrm{C}}\right)$.

Typical results are shown in Fig. 5.

Figure 5 shows that the effect of $\mu$ is very much less dramatic during unloading. The "ideal" (dotted) curve for unloading from $h=-\infty$ sets the maximum possible tension (the pull-off force): here, for $Z=0.4$, equal to 
Fig. 4 Modification of the Fuller and Tabor approach curve by jumping-on due to van der Waals forces. Although it is often claimed that JKR theory holds whenever the Tabor parameter $\mu$ exceeds 5 , loading curves depend strongly on its value even when $\mu>5$

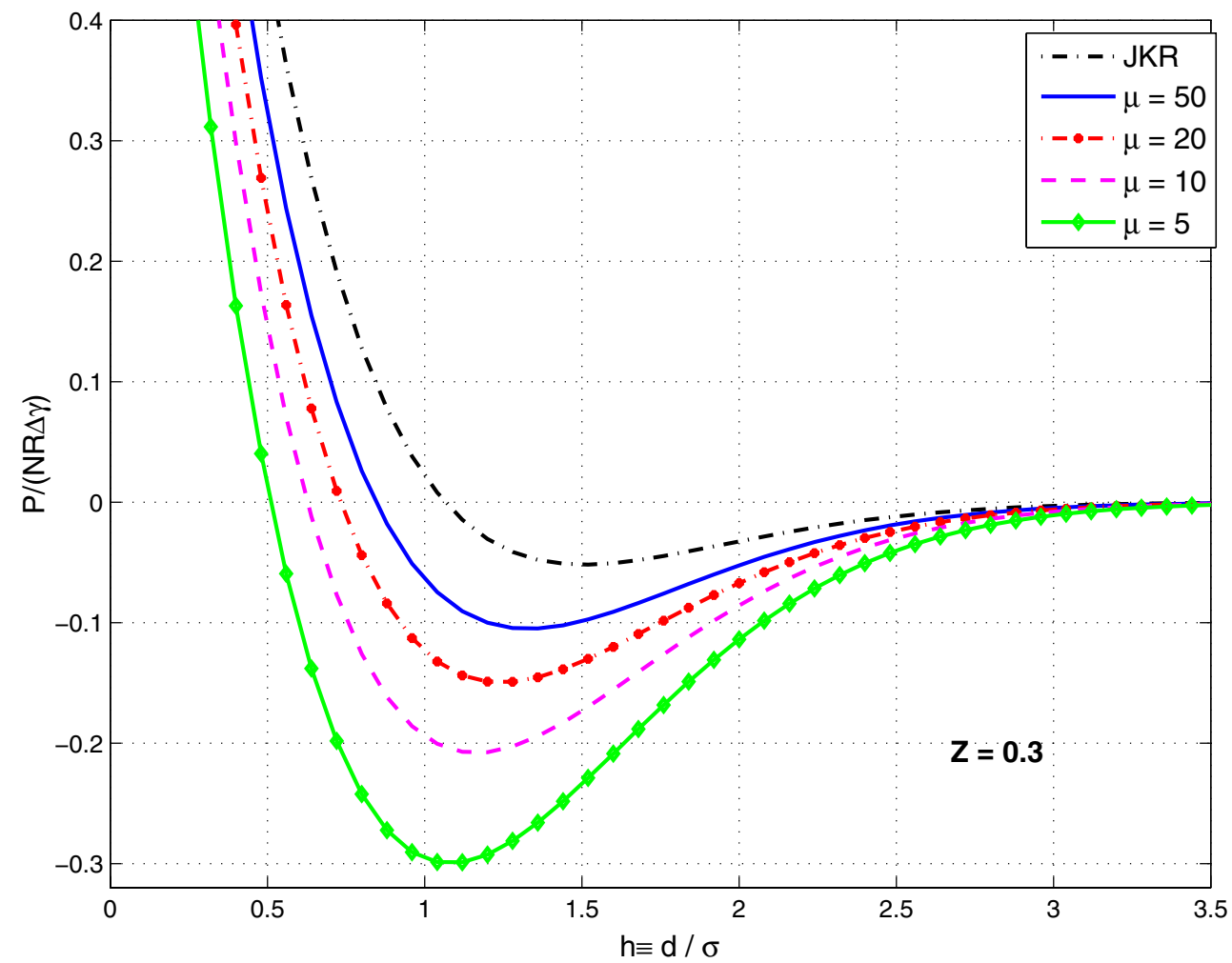

Fig. 5 Loading and unloading curves showing effect of $\mu$. The black dotted curve is the idealised curve for unloading from $h=-\infty$, and all real unloading curves run into it, usually before or close to its minimum, so that the pull-off force is little affected. Note that for $Z=0.4$, zero load is only achievable with $h>0$ when $\mu>10$

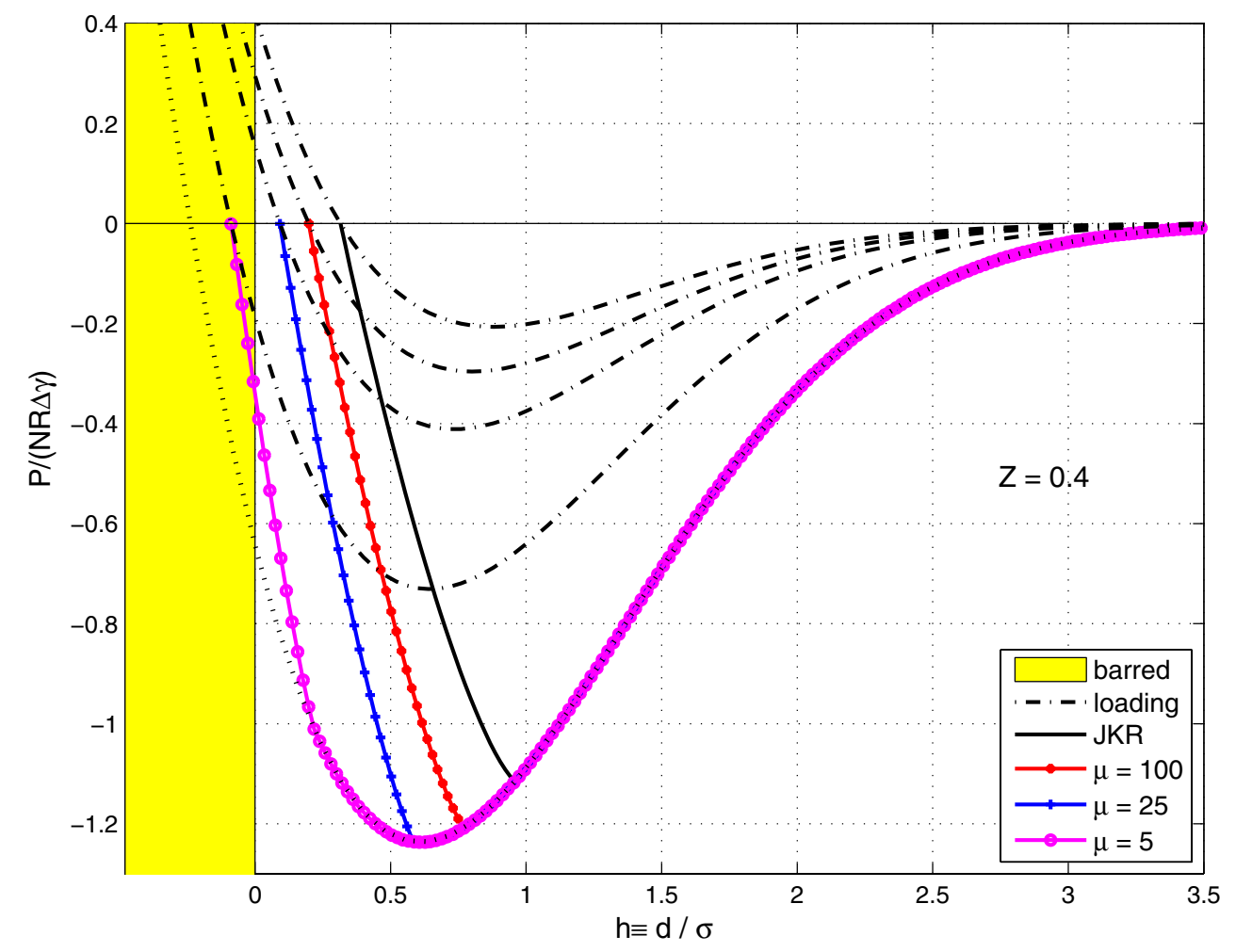

1.24NR $\Delta \gamma$. Whether this is attained depends on where unloading starts from, but, again for $Z=0.4$, the maximum could be reached for all values of $\mu$ by unloading from $h=0$. But for unloading from zero load, the maximum would not be reached for JKR $(\mu=\infty)$ conditions, 


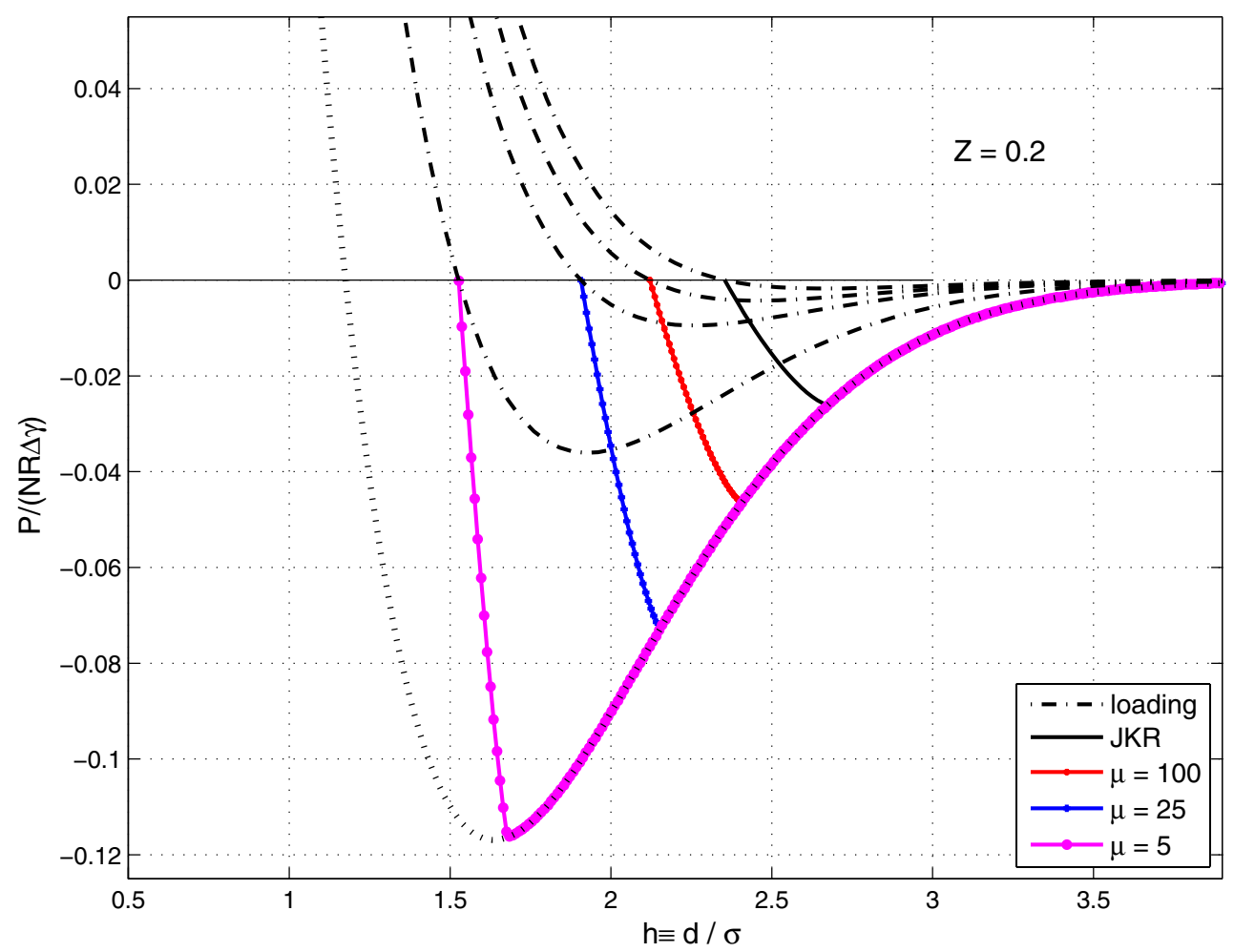

Fig. 6 Loading and unloading curves: $Z=0.2$. For the smaller value of the adhesion parameter the results are very different: now when unloading from zero load, the pull-off force is strongly dependent on the Tabor parameter

but would be reached for $\mu=20$, and, if we trust results from $h<0$, for $\mu=5$ also.

\subsection{Relation Between $\mathrm{Z}$ and $\mu$}

While the two parameters $Z$ and $\mu$ are certainly independent, we note that $Z / \mu=\varepsilon / \sigma$, and there will be few surfaces where the surface roughness $\sigma$ is not an order of magnitude greater than the range of action of the surface forces $\varepsilon$. Thus, for $Z$ in the range $0.2 \leq Z \leq 0.4$, we should restrict ourselves to $\mu \approx 100$ for traditional engineering surfaces, but allow lower values in nanotribology.

Figure 6 shows that even with the restriction that $\mu / Z$ should be large, the pull-off force for $\mu=100$ can be double that for JKR conditions.

\subsubsection{An Upper Bound for the Pull-Off Force}

It is clear that unloading, both for "pure" JKR contacts and when including the effect of early jumping-on when $\mu$ is finite, consists of a transition curve from the unloading point followed by traversing the "ideal" curve: that found by believing the GW model of surface roughness beyond its reasonable limit and unloading from $h=-\infty$. Under many conditions, the ideal curve will be joined before, or near, its maximum, as shown in Fig. 5, though clearly not in Fig. 6. The maximum of the ideal curve therefore provides a safe upper bound for the pull-off force, and this will often also be a useful estimate. Figure 7 shows how it depends on the adhesion index $Z$.

\section{Hysteresis Loss}

The abstract of a recent (otherwise admirable) paper starts:

In experiments that involve contact with adhesion between two surfaces, as found in atomic force microscopy or nanoindentation, two distinct contact force $(P)$ $v s$. indentation-depth $(h)$ curves are often measured depending on whether the indenter moves towards or away from the sample. The origin of this hysteresis is not well understood and is often attributed to moisture, plasticity or viscoelasticity

The origins noted above are very real, but Fig. 2 shows that the classical Fuller and Tabor model certainly provides 


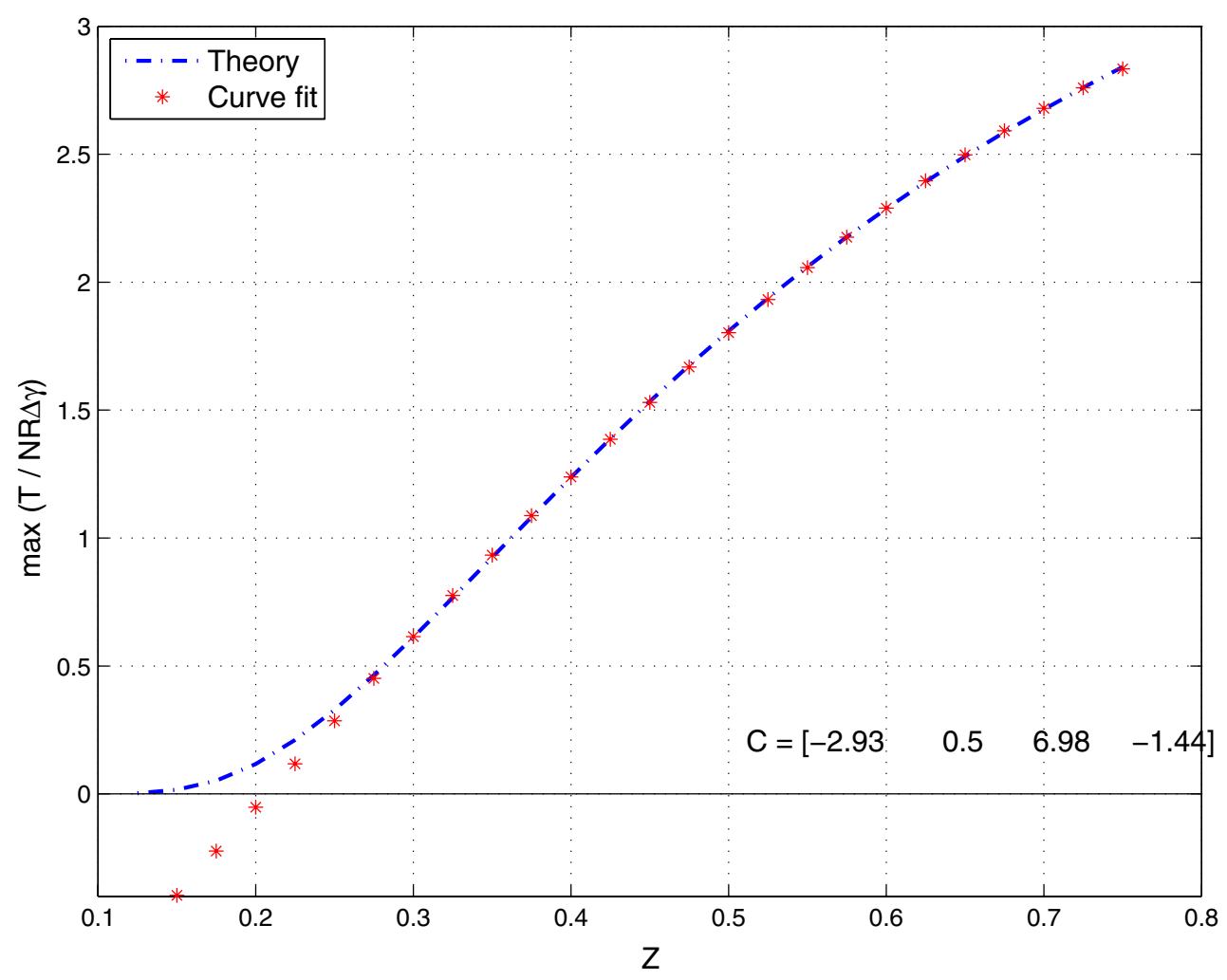

Fig. 7 Upper bounds for the pull-off force. The upper bound for unloading is not affected by the Tabor parameter. $C$ are the coefficients of the curve-fit equation: $\hat{T}=-2.93 Z^{3}+0.5 Z^{2}+6.98 Z-1.44$

yet another origin. It seems possible that Maugis $[12,13]$ by plotting, without comment, only the F\&T unloading curves, may have diverted attention from this effect.

The natural way to calculate the hysteresis energy loss is to find the area between the loading and unloading curves, as indicated in Fig. 8. But Ciavarella et al. [5] point out that there is a more direct way. When we consider the cycle at a single contact, as depicted in Fig. 3, it is clear that only the area $\mathrm{ABCD}$ is relevant to the hysteresis loss: however far beyond point $\mathrm{B}$ the loading is taken, unloading back to point $B$ is along exactly the same path and involves no energy loss. The energy loss $(U)$ contributed by such a contact can be calculated and will be the same for any asperity which makes contact: so the total hysteresis loss will be $n \cdot U$, so may be found without computing the load/approach curves: all that is needed is to determine the maximum number $(n)$ of asperity contacts during the cycle. And this, of course, requires only the loading curve to be determined.

The method of course is valid for a JKR contact, but the early jumping-on when $\mu$ is finite leads to substantially smaller values of $U$. Note that $U$ is a property of an individual contact, so its value depends only on $\mu$ and is independent of $Z$.

\subsection{Hysteresis with the DMT Model?}

Numerical calculations like those resulting in Fig. 3 find that as the Tabor number decreases, the curve between points $\mathrm{C}$ and $\mathrm{D}$ becomes straighter, until when $\mu \approx 0.7$ the re-entry disappears, and there will be no jumping-on and no hysteresis loss for $\mu$ below this value. If, as frequently claimed, the DMT theory is the low $\mu$ limit of such analyses, it therefore predicts no energy loss. However, what is usually meant by the term "DMT" is a gloss on the theory, due to Maugis [4]. Observing that according to the original "thermodynamic" method the adhesive force increases to $2 \pi R \Delta \gamma$ as the contact radius decreases to zero, while according to the later (preferred) "force" method it decreases to this value (see [14]), Maugis ignored both calculations and assumed it to be constant, and so obtained his well-known "DMT equations", which in the present notation are $\hat{\alpha}=\hat{a}^{2} ; \hat{P}=\frac{4}{3} \hat{a}^{3}-2 \pi$. However, it seems clear from Maugis [12] that he regards the adhesion force as dropping abruptly to zero when there is no contact. This seems somewhat drastic: more plausible is to revert to Derjaguin's early calculation [15] and take the adhesion force when the minimum gap is $h_{0}$ to be $T=(2 \pi / 3) R \Delta \gamma\left[4\left(\frac{\varepsilon}{h_{0}+\varepsilon}\right)^{2}-\left(\frac{\varepsilon}{h_{0}+\varepsilon}\right)^{8}\right]$ (smoothly joining on 


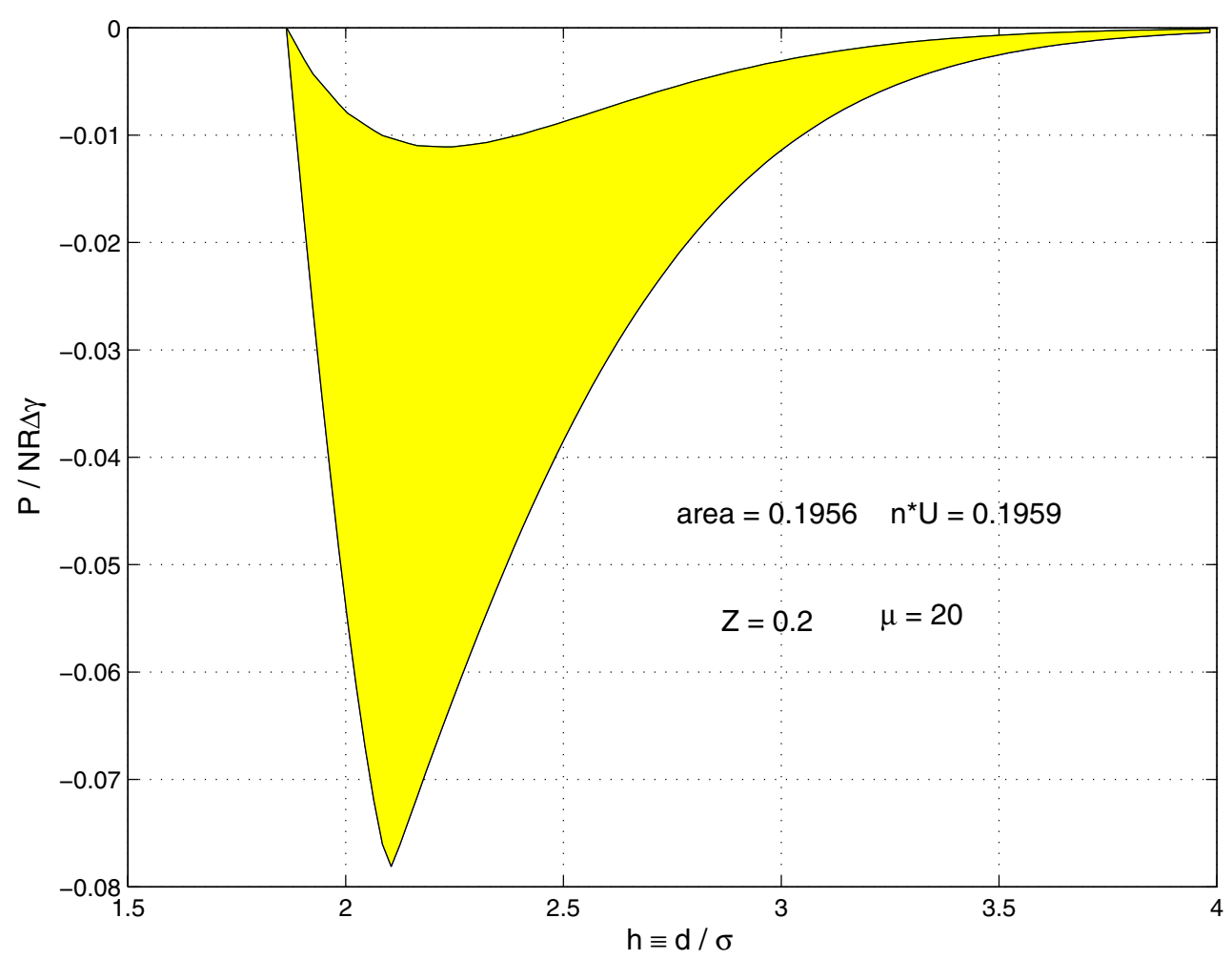

Fig. 8 Hysteresis loss, found by the two methods. Numerical integration of the area between the loading and unloading curves (yellow) gave the energy loss $\hat{U}=0.1956$. Multiplying the unit hysteresis

Table 1 Unit hysteresis loss $\hat{U} \equiv U \cdot\left(E^{* 2} / \Delta \gamma^{5} R^{2}\right)^{1 / 3}$

\begin{tabular}{llllll}
\hline$\mu$ & $\infty(\mathrm{JKR})$ & 100 & 50 & 20 & 5 \\
$\hat{U}$ & 7.092 & 6.319 & 5.954 & 5.209 & 3.216 \\
\hline
\end{tabular}

to $T=2 \pi R \Delta \gamma$ for $\left.h_{0}<0\right)$. This will alter the load/approach curves somewhat: but there seems no reason why this adhesion force should not act both during loading and unloading, and so the conclusion is the same: on the DMT model there will be no hysteresis energy loss.

\section{Behaviour of a Nayak (Random Field) Surface}

Nayak, extending the work of Longuet-Higgins, gave an analysis [16] of the statistical properties of a rough surface as represented by a random field. The surface is specified by three parameters, $m_{0}, m_{2}, m_{4}$, the zero, second and fourth moments of the profile power spectral density, or equivalently the mean square height, slope and curvature $\sigma^{2}, \sigma_{m}^{2}$ $\sigma_{\kappa}^{2}$ of a profile. Many properties depend, except for a scaling factor, only on the parameter $\alpha \equiv m_{0} m_{4} / m_{2}^{2}$. Famously, Bush et al. [17] studied the elastic contact of such a surface loss (Table 1) by the number of contacts as unloading began gave $\hat{U}=0.1959$ (Color figure online)

(the BGT model), though Greenwood [18] and Carbone and Bottiglione [19] corrected their results. More relevantly, they showed that for non-adhesive contacts there is little error in treating the ellipsoidal summits as paraboloids ${ }^{4}$ : the important feature is the variation of the mean asperity curvature with height. It is hoped the same is true for adhesive contacts.

However, from Nayak's analysis, or extending Greenwood [18], although the ensemble has a non-Gaussian height distribution with the mean summit curvature varying with height, it is known ${ }^{5}$ that summits of a given mean curvature have a Gaussian height distribution, but with different mean heights. Specifically, if we scale all heights by the rms height $\sigma \equiv \sqrt{m_{0}}$ and all curvatures by the rms curvature $\sigma_{\kappa} \equiv \sqrt{m_{4}}$, it is known that summits of a given mean curvature $s \equiv \kappa / \sigma_{\kappa}$ have a Gaussian height distribution with mean height $\bar{\varsigma} \equiv \bar{z} / \sigma=3 s / 2 \sqrt{\alpha}$ and standard deviation $\sigma \sqrt{1-3 /(2 \alpha)}$, the standard deviation remarkably (and conveniently!) being the same for all summit curvatures. The density of summits with curvature $s \sigma_{\kappa}$ is

$P(s)=\frac{3}{2 \sqrt{\pi}}\left[\left(3 s^{2}-2\right) \exp \left(-(3 / 4) s^{2}\right)+2 \exp \left(-(9 / 4) s^{2}\right)\right]$,

${ }^{4}$ And Greenwood [18] argues that the summits are not very elliptical.

5 Implicit in Nayak's equations, but first stated explicitly by Whitehouse and Phillips [20]. 
Fig. 9 Results for Nayak surfaces with JKR adhesion. Loading curves: chain-dotted. Unloading curves: continuous. Although the results are qualitatively the same as for a GW surface, the detailed shapes differ

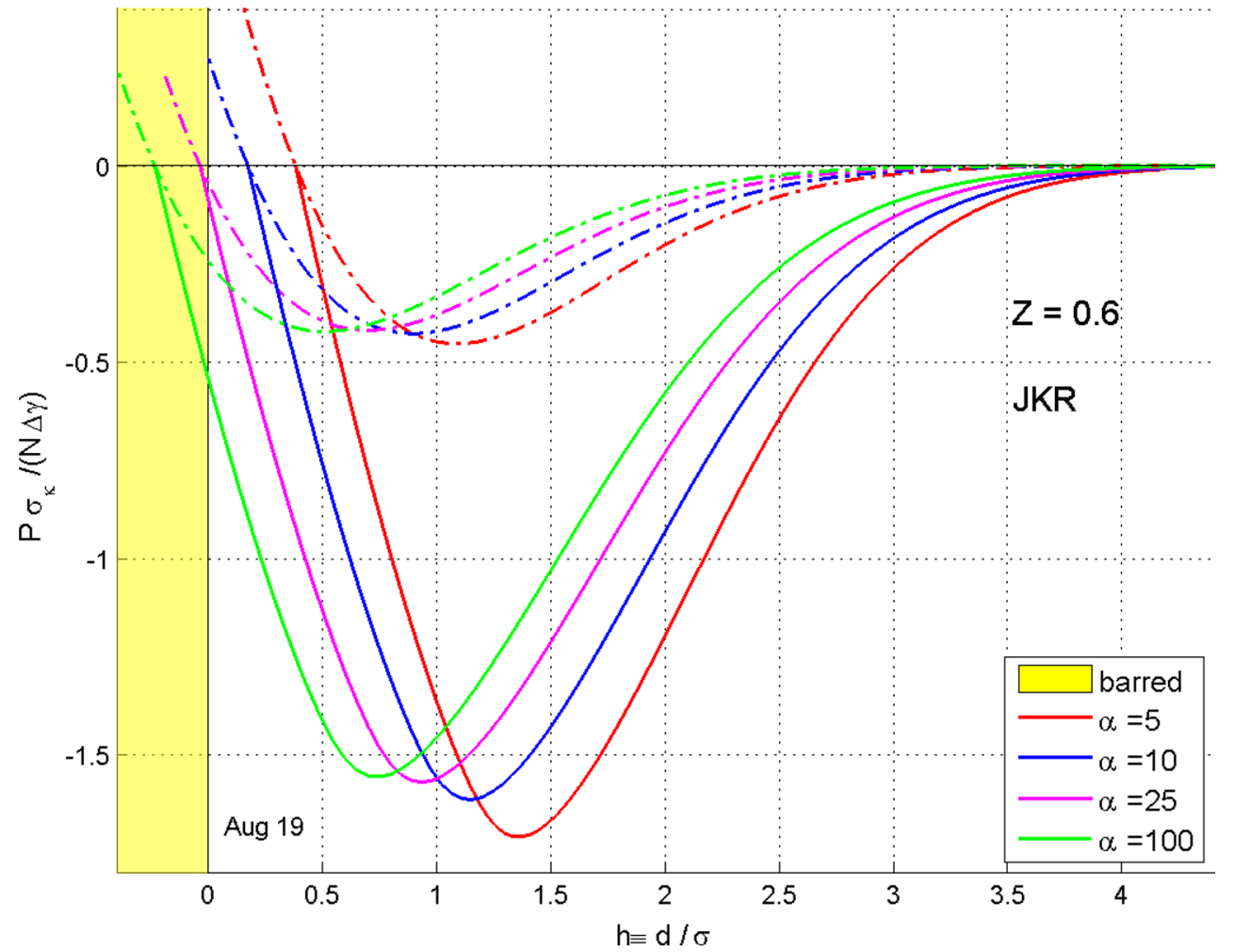

the important range being from $s=0.5$ to $s=3$ : Nayak shows that $\bar{s}=8 / 3 \sqrt{\pi} \approx 1.5045$.

Thus, to analyse the adhesive behaviour of a Nayak surface, we may simply repeat the analysis for a GW surface many times, for a range of summit curvatures (and so with different adhesion indices $Z_{i}(s)$ ), all with the same standard deviation $\sqrt{1-3 /(2 \alpha)}$ but with the appropriate means $3 s / 2 \sqrt{\alpha}$, and combine them in the correct proportions.

We cannot of course use the variable, local adhesion index $Z_{i}(s)$ in the presentation of the results: so the definition of the adhesion index is modified to a standard value $Z \equiv\left(\Delta \gamma^{2} \sigma_{\kappa} / E^{* 2} \sigma^{3}\right)^{1 / 3}\left[\sigma_{\kappa} \equiv \sqrt{m_{4}}, \sigma \equiv \sqrt{m_{0}}\right]$. Then for each subsidiary calculation, we use $Z_{i}(s) \equiv Z / s^{1 / 3}$.

The Tabor parameter must correspondingly be redefined as $\mu \equiv\left(\frac{\Delta \gamma^{2}}{\sigma_{\kappa} E^{* 2} \varepsilon^{3}}\right)^{1 / 3}$, and local values $\mu_{i}(s)$ used in the subsidiary calculations. (And we note that each will yield $\hat{P}=\frac{P s \sigma_{\kappa}}{\Delta \gamma}$, which must be divided by $s$ before combining.)

Figure 9 shows that the results of using the Nayak model of surface roughness are only slightly different. The pattern is clear: as Nayak's parameter $\alpha$ increases, the zero load point requires steadily closer approach, while the pull-off force steadily increases. But the whole pattern is shifted into a more believable range of summit heights, so that a slightly larger value of the adhesion index $(Z=0.6)$ can be studied, and larger adhesion forces achieved. As $\alpha$ increases, the magnitude of the pull-off force somewhat reduces (Fig. 10).
As for the simple GW model of surface roughness, using the extended JKR model slightly increases the pull-off force, the effect saturating rather soon, with the pull-off force for $\mu=100$ being little less than for smaller values of $\mu$. In contrast—but again as found for the GW model — the loading curves have very considerably increased forces. The result is a substantial reduction in the hysteresis loss: the loss here for $\mu=5$ being perhaps one-third of the JKR value.

\subsection{Discussion: Definition of $Z$}

Perhaps for comparison with the previous results, we should have chosen $Z_{\kappa} \equiv\left(\Delta \gamma^{2} / E^{* 2} \bar{\kappa} \sigma^{3}\right)^{1 / 3}$, replacing $R$ by $1 / \bar{\kappa}$ as suggested by McCool [21], and widely followed. But this is highly speculative: certainly the mean radius of curvature is not the reciprocal of the mean curvature $\bar{\kappa}$. [First lesson in probability theory: the reciprocal of the mean of $x$ rarely equals the mean of the reciprocal of $x$ ! A rather appropriate example is that the mean peak curvature multiplied by the mean peak radius of curvature equals $(\pi / 2)$.] But perhaps a greater problem is that Nayak's mean summit curvature is the mean for all the summits, including the contribution from the perhaps $50 \%$ of summits lying below the summit mean plane: and summit curvature is known to increase strongly with height!

We note that Nayak shows that $\bar{\kappa} \equiv(8 / 3 \sqrt{\pi}) \sigma_{\kappa}$, so that $Z_{\sigma_{\kappa}}$ and $Z_{\bar{\kappa}}$ differ only by a factor $1.504^{1 / 3} \approx 1.15$. 
Fig. 10 Effect of early jumping-on for a Nayak surface. As found for a GW surface, the early jumping-on for finite values of $\mu$ has a large effect on the loading curves (chain-dotted), but a rather small effect on the unloading curves (continuous)

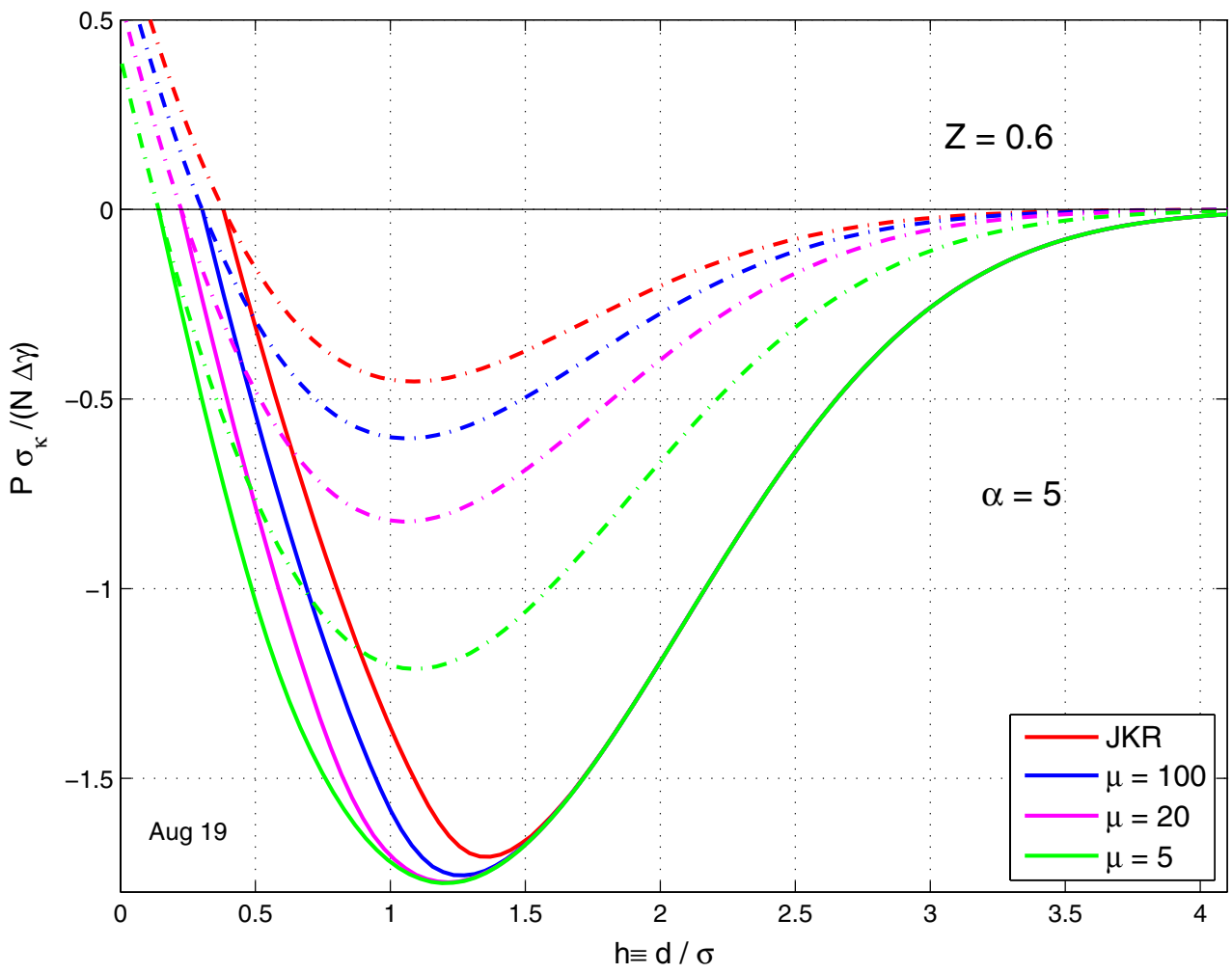

\section{Discussion}

With the progress in the understanding of surface roughness in the last 50 years, it is no longer possible to take the predictions from asperity theories as serious approximations to real behaviour. The adhesion forces calculated above are the values of $P / N R \Delta \gamma$ or $P \sigma_{\kappa} / N \Delta \gamma$, but what is $N$ ? There may indeed be a countable number of summits on a worn surface (and indeed on a Nayak surface the summit density is known to be $\frac{1}{6 \pi \sqrt{3}} \frac{m_{4}}{m_{2}}$ [16]), but what proportion of these can be regarded as independent asperities? Almost zero on a fractal surface! So was this a pointless exercise? The author believes not: for the asperity approach leads naturally to an understanding of the difference between loading and unloading, and so to why there is hysteresis: it does not require viscoelasticity or process-dependent surface energy: these are additional effects. Individual contact areas may not obey the JKR theory, but they still form and separate under different rules. Adhesion is the outcome of a battle between tensile contacts and compressive contacts: a feature which seems to be overlooked in some grand numerical analyses of adhesive contact.

Another, related, lesson from asperity theories is that the tempting experimental procedure of slightly overloading the contact to manage slightly viscoelastic response will bring into contact areas which "should not" have made contact, and so may give incorrect unloading curves. This will not always be serious: in a number of calculations the pull-off force has proved to be almost, or completely, independent of the point at which unloading starts, although the initial parts of the curve certainly do differ.

\subsection{GW Versus Nayak}

Are the results of the two surface roughness models significantly different? Quite possibly not: for while there is no doubt that larger values of the adhesion parameter may be used with a Nayak surface than with a GW surface without violating our " $h>0$ " rule, this may simply be the result of the inevitably different definitions of $Z$ (and indeed of $\mu$ and $\hat{P})$. The numerical factor relating the mean asperity radius of curvature $R$ to $1 / \sigma_{\kappa}$ will certainly not be the same when contact mainly involves asperities with heights between $2 \sigma$ and $3 \sigma$, as usual in non-adhesive contact, as it is when much lower asperities are involved. Perhaps in the end we can only say that adhesion is controlled by the ratio of $\left(\Delta \gamma / E^{*}\right)$ to a length related to the surface topography but involving both the amplitude of the height variation and some measure of the smoothness. 


\section{Conclusion}

The study of adhesion using an asperity model gives valuable qualitative insight into the adhesion of rough surfaces: it brings out that a quasi-Tabor parameter will be significant as well as an adhesion parameter and that their ratio will be linked to the ratio $\sigma / \varepsilon$ : of a surface roughness amplitude to the range of action of the surface forces.

Open Access This article is distributed under the terms of the Creative Commons Attribution 4.0 International License (http://creativecommons.org/licenses/by/4.0/), which permits unrestricted use, distribution, and reproduction in any medium, provided you give appropriate credit to the original author(s) and the source, provide a link to the Creative Commons license, and indicate if changes were made.

\section{Appendix 1: CGB Notation}

The use of separation rather than compression seems to be inconvenient here: so we reverse the signs of $\delta$ and " $T$ " to make the equations closer to the Hertz equations as used in the GW theory: $\hat{P}=\frac{P}{R \Delta \gamma} ; \hat{\delta}=\frac{\beta^{2} \delta}{R} ; \hat{a}=\frac{\beta a}{R} ;$ where $\beta \equiv\left(\frac{E^{*} R}{\Delta \gamma}\right)^{1 / 3}=\sqrt{\frac{R}{\mu \varepsilon}}$.

With this notation, avoiding the numerical factors introduced by Maugis to simplify the JKR equations, these become $^{6} \hat{\delta}=\stackrel{\check{a}}{2}-\sqrt{2 \pi \hat{a}} ; \hat{P}=(4 / 3) \hat{a}^{3}-\sqrt{8 \pi \hat{a}^{3}}$ : a minor complication for the gain of avoiding numerical factors in the relations between the dimensionless and the physical variables.

We note that the pull-off force is $-\hat{P}_{m}=\hat{T}_{m}=3 \pi / 2$ and occurs when $\hat{a}=\hat{a}_{m} \equiv(9 \pi / 8)^{1 / 3}$ and $\hat{\delta}=\hat{\delta}_{m} \equiv-(1 / 4)\left(3 \pi^{2}\right)^{1 / 3} \approx-0.773$.

However here we are displacement controlled, and separation occurs when $(-\delta)$ is a maximum: at $\hat{a}=\hat{a}_{c} \equiv(\pi / 8)^{1 / 3}$, $\hat{\delta}=\hat{\delta}_{c} \equiv-(3 / 4) \pi^{2 / 3} \approx-1.609$. Then $P_{m}=-5 \pi / 6$.

\section{Appendix 2: Numerical Evaluations}

Fuller and Tabor [1] invert the JKR equations to obtain an explicit (curve-fit) function $f(\hat{\delta})$. Here we use a mathematically more elegant method suggested by Prof. J R Barber: we note that the JKR equations give the load and approach as explicit functions of the contact radius $\hat{a}$ and that the $\hat{\delta}(\hat{a})$ relation is monotonic ${ }^{7}$ : so we may change the integration variable to $\hat{a}$. We have $\frac{\mathrm{d} \hat{\delta}}{\mathrm{d} \hat{a}}=2 \hat{a}-\frac{\sqrt{2 \pi}}{2 \sqrt{\hat{a}}}$ so that

\footnotetext{
${ }^{6}$ So $\delta$ is again a compression and $P$ a compression force.
}

$$
\begin{aligned}
f(\hat{\delta}) \frac{\mathrm{d} \hat{\delta}}{\mathrm{d} \hat{a}} & =\left(\sqrt{8 \pi \hat{a}^{3}}-(4 / 3) \hat{a}^{3}\right)\left(2 \hat{a}-\frac{\sqrt{2 \pi}}{2 \sqrt{\hat{a}}}\right) \\
& =-\hat{a}\left(\sqrt{2 \pi}-(2 / 3) \hat{a}^{3 / 2}\right)\left(\sqrt{2 \pi}-4 \overleftarrow{a}^{3 / 2}\right) .
\end{aligned}
$$

Thus

$$
\begin{gathered}
\hat{P} / N=\frac{-Z}{\sqrt{2 \pi}} \int_{\hat{a}_{0}}^{\infty} \exp \left[-\{h+Z \hat{\delta}\}^{2} / 2\right][2 \pi-(14 / 3) \\
\left.\sqrt{2 \pi} \hat{a}^{3 / 2}+(8 / 3) \hat{a}^{3}\right] \hat{a} \mathrm{~d} \hat{a}
\end{gathered}
$$

This is now a straightforward explicit numerical integration.

However, note that the lower limit of the integral is not zero but, for loading, $\hat{a}_{0}=(2 \pi)^{1 / 3}$, for in the JKR theory immediately on contact the contact radius jumps to this value. For finite values of $\mu$, where we assume that the jump into contact occurs at $\hat{\delta}_{A}=\frac{1}{\mu}-\frac{2.641}{\mu^{4 / 7}}$, we need to find the value of $a_{B}$ : this is conveniently done by rewriting the JKR equation $\hat{\delta}=\grave{a}-\sqrt{2 \pi \hat{a}}$ as a quartic equation in $t \equiv \sqrt{\hat{a}}$ : $\left[t^{4}-t \sqrt{2 \pi}-\hat{\delta}_{A}=0\right]$; this is solved by the MATLAB routine "roots" and the larger real root is then selected to give $\hat{a}_{B}=t^{2}$.

For unloading in the JKR case the lower 1 imit is $\quad \hat{\delta}_{0} \equiv \min \left(\left\{\left(h_{1}-h\right) / Z\right\}, \hat{\delta}_{c}\right), \quad$ where $\hat{\delta}_{c} \equiv-(3 / 4) \pi^{2 / 3} \approx-1.609$ and correspondingly $\hat{a}_{c} \equiv(\pi / 8)^{1 / 3} \approx 0.732$. For finite values of $\mu$ the lower limit is modified to $\hat{\delta}_{0}=\max \left\{\left(h_{1}-h\right) / Z, \hat{\delta}_{c}\right\}$ and the corresponding value of $a_{c}$ found as above by solving the quartic equation.

To find the unit hysteresis loss (by the proposed approximation), it is only necessary to find the area under the relevant section of the JKR curve: this is

$\hat{U} \equiv U \cdot\left(E^{* 2} / \Delta \gamma^{5} R^{2}\right)^{1 / 3}=\left[\frac{8}{15} \hat{a}^{5}+\pi \hat{a}^{2}-(4 / 3) \sqrt{2 \pi} \cdot \hat{a}^{7 / 2}\right]_{a_{B}}^{a_{C}}$

\section{Appendic C: Details Using the Nayak Theory}

The definition of the adhesion index is here modified to $Z \equiv\left(\Delta \gamma^{2} / E^{* 2} \sigma_{\kappa} \sigma^{3}\right)^{1 / 3}$ where $\sigma_{\kappa} \equiv \sqrt{m_{4}}$ and $\sigma \equiv \sqrt{m_{0}}$ : then for each curvature value during the calculation we need to use a local $Z_{i} \equiv Z / f s^{1 / 3}$, where we have written $f$ for the factor $\sqrt{1-3 /(2 \alpha)}$ so that the height standard deviation is $f \sigma$. Similarly, the load parameter becomes $\hat{P} \equiv P \sigma_{\kappa} / \Delta \gamma$, but the separate calculations yield $\hat{P}_{i} \equiv P\left(s \sigma_{\kappa}\right) / \Delta \gamma$.

Other quantities also need redefinition: the Tabor parameter becomes $\mu \equiv\left(\frac{\Delta \gamma^{2}}{\sigma_{\kappa} E^{* 2} \varepsilon^{3}}\right)^{1 / 3}$, and the appropriate local value $\mu_{i}=\mu / s^{1 / 3}$ must be used for each summit curvature.

\footnotetext{
${ }^{7}$ In the practical range: only at the "fixed grips" jump-off point do we get $\mathrm{d} \delta / \mathrm{d} a=0$.
} 


\section{References}

1. Fuller, K.N.G., Tabor, D.: The effect of surface roughness on the adhesion of elastic solids. Proc. Roy. Soc. Lond. A345, 327-342 (1975)

2. Greenwood, J.A., Williamson, J.B.P.: Contact of nominally flat rough surfaces. Proc. R. Soc. Lond. A295, 300 (1966)

3. Johnson, K.L., Kendall, K., Roberts, A.D.: Surface energy and the contact of elastic solids. Proc. R. Soc. Lond. A 324, 301-313 (1971)

4. Maugis, D.: Adhesion of spheres: the JKR-DMT transition using a Dugdale model. J. Colloid Interface Sci. 150, 242-269 (1992)

5. Ciavarella, M., Greenwood, J.A., Barber, J.R.: Effect of the Tabor parameter on hysteresis losses during adhesive contact. J. Mech. Phys. Solids 98, 236-244 (2017)

6. Tabor, D.: Surface forces and surface interactions. J. Colloid Interface Sci. 58, 2-13 (1977)

7. Muller, V.M., Yuschenko, V.S., Derjaguin, B.V.: On the influence of molecular forces on the deformation of an elastic sphere and its sticking to a rigid plane. J. Colloid Interface Sci. 77, 91 (1980)

8. Greenwood, J.A.: Adhesion of elastic spheres. Proc. R. Soc. Lond. A453, 1277-1297 (1997)

9. Feng, J.Q.: Contact behavior of spherical elastic particles: a computational study of particle adhesion and deformations. Colloids Surf. 172, 175-198 (2000)

10. Feng, J.Q.: Adhesive contact of elastically deformable spheres: a computational study of pull-off force and contact radius. J. Colloid Interface Sci. 238, 318-323 (2001)
11. Wu, J.-J.: The jump-to-contact distance in atomic force microscopy measurement. J. Adhes. 86, 1071-1085 (2010)

12. Maugis, D.: On the contact and adhesion of rough surfaces. $\mathbf{J}$ Adhes. Sci. Technol. 10(2), 161-175 (1996)

13. Maugis, D.: Contact, Adhesion and Rupture of Elastic Solids. Springer, New York (2000)

14. Pashley, M.D.: Further considerations of the DMT model for elastic contact. Colloids Surf. 12, 69-77 (1984)

15. Derjaguin, B.V.: Theorie des Anhaftens kleiner Teilchen. KolloidZeitschrift 69(2), 155-164 (1934)

16. Nayak, P.R.: Random process model of rough surfaces. ASME J. Lubr. Technol. 93, 398-407 (1971)

17. Bush, A.W., Gibson, R.D., Thomas, T.R.: The elastic contact of a rough surface. Wear 35, 87-111 (1975)

18. Greenwood, J.A.: A simplified elliptic model of rough surface contact. Wear 261, 191-200 (2006)

19. Carbone, G., Bottiglione, F.: Asperity contact theories: do they predict linearity between contact area and load? J. Mech. Phys. Solids 56, 2555-2572 (2008)

20. Whitehouse, D.J., Phillips, M.J.: Two dimensional discrete properties of random surfaces. Philos. Trans. Roy. Soc. A305, 441-468 (1982)

21. McCool, J.I.: Comparison of models for the contact of rough surfaces. WEAR 107, 37-60 (1986) 